\title{
Asymptotic analysis of numerical steepest descent with path approximations
}

\author{
Andreas Asheim and Daan Huybrechs
}

Report TW 536, March 2009

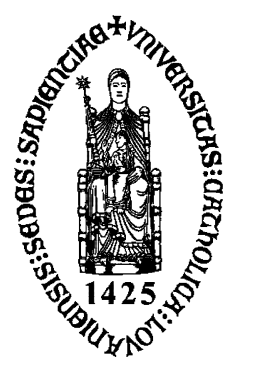

Katholieke Universiteit Leuven Department of Computer Science

Celestijnenlaan 200A - B-3001 Heverlee (Belgium) 


\title{
Asymptotic analysis of numerical steepest descent with path approximations
}

\author{
Andreas Asheim and Daan Huybrechs \\ Report TW 536, March 2009
}

Department of Computer Science, K.U.Leuven

\begin{abstract}
We propose a variant of the numerical method of steepest descent for oscillatory integrals by using a low-cost explicit polynomial approximation of the paths of steepest descent. A loss of asymptotic order is observed, but in the most relevant cases the overall asymptotic order remains higher than a truncated asymptotic expansion at similar computational effort. Theoretical results based on number theory underpinning the mechanisms behind this effect are presented.
\end{abstract}

Keywords : oscillatory integral, steepest descent, numerical integration AMS(MOS) Classification : Primary : 65D30, Secondary : 30E20, 41A60. 


\title{
Asymptotic analysis of numerical steepest descent with path approximations
}

\author{
Andreas Asheim, Daan Huybrechs ${ }^{\dagger}$
}

March 16, 2009

\begin{abstract}
We propose a variant of the numerical method of steepest descent for oscillatory integrals by using a low-cost explicit polynomial approximation of the paths of steepest descent. A loss of asymptotic order is observed, but in the most relevant cases the overall asymptotic order remains higher than a truncated asymptotic expansion at similar computational effort. Theoretical results based on number theory underpinning the mechanisms behind this effect are presented.
\end{abstract}

AMS(MOS) Classification: Primary : 65D30, Secondary : 30E20, 41A60. Keywords: oscillatory quadrature, steepest descent, numerical integration.

\section{Introduction}

Consider a highly oscillatory integral of the form

$$
I[f]=\int_{-1}^{1} f(x) e^{i \omega g(x)} \mathrm{d} x,
$$

where $\omega$ is a large parameter and $f$ and $g$ are smooth functions called the amplitude function and oscillator of the integral respectively. Such integrals, often referred to as Fourier-type integrals, appear in a wide area of applications, e.g., highly oscillatory scattering problems in acoustics, electromagnetics or optics $[5,3,13,2]$. Numerical evaluation of Fourier-type integrals with classical techniques becomes expensive as $\omega$ becomes large, which corresponds to a highly oscillatory integral. Typically, a fixed number of evaluation points per wavelength is required to obtain a fixed accuracy, which makes the computational effort at least linear in $\omega[6]$.

\footnotetext{
*Department of Mathematical Sciences, NTNU, 7491 Trondheim, Norway. E-mail: Andreas.Asheim@math.ntnu.no

${ }^{\dagger}$ Department of Computer Science, KU Leuven, 3001 Leuven, Belgium. E-mail: Daan.Huybrechs@cs.kuleuven.be. This author is a PostDoctoral Fellow of the Research Foundation - Flanders (FWO).
} 
Asymptotic techniques on the other hand yield approximations that become more accurate as $\omega$ increases, making them superior for $\omega$ sufficiently large. One of these techniques, the principle of stationary phase [20, 25], states that $I[f]$ asymptotically depends only on $f$ and $g$ in a set of special points as $\omega \rightarrow \infty$. These points are the endpoints, here $x=-1$ and $x=1$, and stationary points - points where the derivative of $g$ vanishes. At stationary points the integral is locally non-oscillatory. The integral has an asymptotic expansion in inverse powers of $\omega$, with coefficients that depend on the derivatives of $f$ and $g$ at these critical points [15].

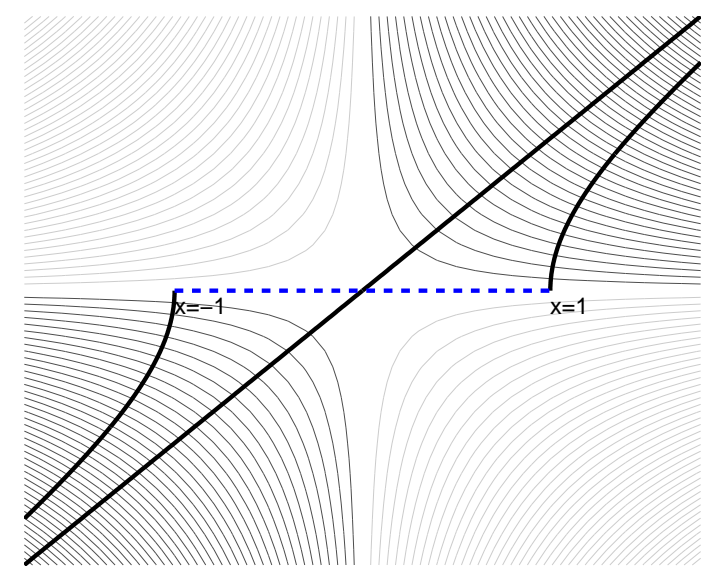

Figure 1: The contours of the imaginary part of the oscillator $g(x)=x^{2}$ in the complex plane and the corresponding paths of steepest descent. Two paths emerge from the endpoints $x=-1$ and $x=1$. They are connected by a path passing through the stationary point at $x=0$.

A set of particularly effective ways of obtaining the contribution from a special point are the saddle point methods $[25,19,8]$. Based on Cauchy's integral theorem, the path of integration can be deformed into the complex plane without changing the value of the integral, provided that $f$ and $g$ are analytic [9]. The method of steepest descent is obtained by following a path where $g$ has a constant real part and increasing imaginary part, which renders the integral (1.1) non-oscillatory and exponentially decreasing. This procedure yields separate paths originating from each special point that typically connect at infinity (see Figure 1 for an illustration). The result is separate contributions corresponding to each special point. Every one of these contributions is a non-oscillatory integral that can be written as

$$
\int_{0}^{\infty} \psi(q) e^{-\omega q^{r}} \mathrm{~d} q,
$$


where $\psi$ is a smooth function, $r=1$ for endpoint contributions, and $r>1$ for stationary points. These integrals are usually treated with standard asymptotic techniques like Watson's Lemma. The larger class of saddle point methods also contains methods that follow other paths with similar characteristics as the steepest descent paths, e.g., Perron's method[25].

The asymptotic expansion of $I[f]$ in general diverges, but it can yield very accurate approximations if $\omega$ is very large. Still, divergence implies that the error is uncontrollable, which is problematic in the context of numerical computations. Recent research has however produced several numerical methods that exhibit convergence. The Filon-type methods $[15,14,16]$ are based on polynomial interpolation of the amplitude $f$ and can deliver errors that are $\mathcal{O}\left(\omega^{-p}\right)$ for any $p$, much like truncated asymptotic expansions, but with controllable error for fixed $\omega$. Filon-type methods require that moments $w_{k}=I\left[x^{k}\right]$ are available, a serious drawback in some cases. Combining asymptotic expansions and Filon-type methods[1] can economise on, but not eliminate the need for moments. Methods that do not rely on moments are the Levin-type methods, due to Levin[18] and extended by Olver[22, 23]. Levin-type methods do not work in the presence of stationary points, but a work-around is provided in [21]. We refer the reader to [11] for a detailed overview of these and other numerical methods.

One of the alternatives is the numerical method of steepest descent [12], which is a numerical adaptation of the above described method of steepest descent. Relying on classical numerical integration methods applied to an exact decomposition of the integral, the numerical method of steepest descent has controllable error wherever the exact decomposition is available, and asymptotic error decay $\mathcal{O}\left(\omega^{-p}\right)$ for any $p$. The paths of steepest descent can however be difficult to compute, as their computation corresponds to solving a non-linear problem that can in practice only be solved iteratively.

The method of this paper is similar in spirit but based on the practical observation that the exact choice of path is not essential. This observation resonates with the theory behind saddle point methods. A Taylor expansion of the path of steepest descent, which can explicitly be derived from a Taylor expansion of the oscillator function $g$, is in many cases sufficient. Iterative methods to solve a non-linear problem can therefore be entirely avoided. We obtain a numerical scheme which is relatively simple to implement and cheap to evaluate. The method exhibits high asymptotic order, and the order is in fact higher than one would get from a truncated asymptotic expansion using exactly the same number of derivatives of $g$.

It is the purpose of this paper to analyse the asymptotic order of the proposed explicit numerical saddle-point method. Unlike the numerical adaptation of the steepest descent method and the other methods for highly oscillatory integrals mentioned above, the asymptotic order does not follow from standard results in asymptotic analysis. A seemingly irregular relation between the number of derivatives of $g$ that are used and the number of 
quadrature points along the approximate paths of steepest descent can only be explained in terms of elementary number theory. The main result of this paper is formulated and proved in $\S 4$ in Theorem 4.3 .

\section{The numerical method of steepest descent}

In this section we give a brief overview of the numerical method of steepest descent. For a more thorough treatment, see [25] for the classical method of steepest descent, and [12] for particularities on the numerical version. In the following, we will for simplicity assume that all paths may extend to infinity, which implies among other things that $f$ and $g$ should be analytic in a sufficiently large portion of the complex plane. We note that this requirement can be significantly relaxed if so desired[10].

\subsection{Paths of steepest descent}

For the oscillatory integral (1.1) the path of steepest descent $h_{x}(p)$ originating from the point $x$ can be found by solving the equation

$$
g\left(h_{x}(p)\right)=g(x)+i p .
$$

Subject to the boundary condition $h_{x}(0)=x$, equation (2.1) is uniquely solvable for small $p$ if $g^{\prime}(x) \neq 0$. Along the path of steepest descent we have $e^{i \omega g\left(h_{x}(p)\right)}=e^{i \omega g(x)} e^{-\omega p}$, which means that the line integral

$$
I\left[f ; h_{x}, P\right]=e^{i \omega g(x)} \int_{0}^{P} f\left(h_{x}(p)\right) h_{x}^{\prime}(p) e^{-\omega p} \mathrm{~d} p,
$$

is non-oscillatory and exponentially decreasing.

Along paths of steepest descent originating from different points, $g_{x}(h(p))$ may have different, constant real parts, hence the paths may never meet. A connecting path must therefore be introduced. If there are no stationary points, the paths may connect at infinity by letting $P \rightarrow \infty$. In that case, the connecting path has no contribution to the value of the integral. In the presence of stationary points in $[-1,1]$ however, the total path must pass through all of these points and their contributions are not negligible.

Any value $\xi \in[-1,1]$ such that $g^{\prime}(\xi)=0$ is called a stationary point. We call $\xi$ a stationary point of order $r-1$ if $g^{(i)}(\xi)=0$ for $i=1,2, \ldots, r-1$, and $g^{(r)}(\xi) \neq 0 .{ }^{1}$ The canonical example is $g(x)=x^{r}$. At a stationary point, equation (2.1) may have several solutions. In particular, if $\xi$ is a stationary point of order $r-1>0$, then there are $r$ different paths, $h_{\xi, j}, j=1, \ldots, r$, emerging from $\xi$. Since the total path passes trough $\xi$ only once, exactly

\footnotetext{
${ }^{1}$ Note that with this definition an endpoint is a stationary point of order 0 .
} 
two of these paths are relevant. We denote these two paths by $h_{\xi, j_{1}}$ and $h_{\xi, j_{2}}$. Each of these paths corresponds to an integral of the form

$$
I\left[f ; h_{\xi, j}, P\right]=e^{i \omega g(\xi)} \int_{0}^{P} f\left(h_{\xi, j}(p)\right) h_{\xi, j}^{\prime}(p) e^{-\omega p} \mathrm{~d} p .
$$

Again, letting $P \rightarrow \infty$ eliminates contributions from connecting paths, with certain assumptions. Writing

$$
I\left[f ; h_{x}\right]=\lim _{P \rightarrow \infty} I\left[f ; h_{x}, P\right],
$$

the integral (1.1) is represented as a sum of contributions

$$
I[f]=I\left[f ; h_{-1}\right]-I\left[f ; h_{\xi_{1}, j_{1}}\right]+I\left[f ; h_{\xi_{1}, j_{2}}\right]+\ldots-I\left[f ; h_{\xi_{n}, j_{1}}\right]+I\left[f ; h_{\xi_{n}, j_{2}}\right]-I\left[f ; h_{1}\right],
$$

where $\xi_{1}, \ldots, \xi_{n}$ are stationary points. We will concentrate on integrals of the type $I[f ; h]$, hereafter referred to as steepest descent integrals.

\subsection{Numerical evaluation of steepest descent integrals}

Steepest descent integrals can be approximated efficiently with Gaussian quadrature. This is the observation behind the numerical method of steepest descent, which we shall briefly explain here.

For convenience, we introduce the notation

$$
f_{x}(p)=f\left(h_{x}(p)\right) h_{x}^{\prime}(p) .
$$

The contribution from an endpoint becomes

$$
I\left[f ; h_{x}\right]=e^{i \omega g(x)} \int_{0}^{\infty} f_{x}(p) e^{-\omega p} \mathrm{~d} p=\frac{e^{i \omega g(x)}}{\omega} \int_{0}^{\infty} f_{x}\left(\frac{t}{\omega}\right) e^{-t} \mathrm{~d} t .
$$

Since $f_{x}(t / \omega)$ is smooth, this integral can be computed efficiently with classical Gauss-Laguerre quadrature for the weight function $e^{-t}[6]$. Applying an $n$-point quadrature yields an approximation with error $\mathcal{O}\left(\omega^{-2 n-1}\right)$ [12]. Truncating the asymptotic expansion after $n$ terms yields only $\mathcal{O}\left(\omega^{-n-1}\right)$ asymptotic error, but requires the same number of evaluations of $f$.

For the contribution from a stationary point things are a little different. When $\xi$ is a stationary point of order $r-1>0, h_{\xi}(p)$ behaves as $p^{1 / r}$ near $p=0$ and $h_{\xi}^{\prime}(p)$ has a $p^{-(r-1) / r}$ singularity [9]. This singularity can be canceled by the substitution $p=q^{r}$. The contribution is now written

$$
\begin{aligned}
I\left[f ; h_{\xi}\right] & =r e^{i \omega g(\xi)} \int_{0}^{\infty} f_{\xi}\left(q^{r}\right) q^{r-1} e^{-\omega q^{r}} \mathrm{~d} q \\
& =\frac{r e^{i \omega g(\xi)}}{\omega} \int_{0}^{\infty} f_{\xi}\left(\frac{t^{r}}{\omega}\right) t^{r-1} e^{-t^{r}} \mathrm{~d} t .
\end{aligned}
$$

This is an integral of the form (1.2). Since $f_{\xi}\left(\frac{t^{r}}{\omega}\right) t^{r-1}$ is a smooth function, the integral can be efficiently approximated by Gaussian quadrature with 
weight function $e^{-t^{r}}$. We note that it may be beneficial to merge the two contributions from a stationary point into a single integral over the whole real line. For example, in the case of a first order stationary point $(r=2)$, classical Gauss-Hermite quadrature can be applied[7]. In this exposition, however, we will only work with integrals on the half-space.

The result of applying an $n$-point Gaussian quadrature leads to an approximation with an error which is $\mathcal{O}\left(\omega^{-(2 n+1) / r}\right)$ as $\omega \rightarrow \infty$ [7]. In contrast, truncating the asymptotic expansion after $n$ terms yields only $\mathcal{O}\left(\omega^{-(n+1) / r}\right)$ asymptotic error, but requires the same number of evaluations of $f$.

\section{A numerical saddle point method}

Finding the path of steepest descent means solving equation (2.1). This is a non-linear equation and solving it amounts to computing the inverse function $g^{-1}$, which in practical applications may be difficult to achieve. The rationale in this section is that in many cases it is sufficient to have only a rough approximation of the exact steepest descent path. If not, then the rough approximation is still useful as a starting value for, e.g., Newton iterations to solve the non-linear equation numerically.

Here, we obtain a local approximation of the path by means of its Taylor series around $x$. Only derivatives of $g$ at $x$ are used to construct this approximation. This approximate path may diverge away from the actual steepest descent path deep into the complex plane. However, this is not a problem in practice provided $\omega$ is large: because the quadrature points cluster towards $x$ as $\omega$ grows, as can be seen from equations (2.2) and (2.3), a good approximation close to the real axis is generally sufficient.

\subsection{Local paths at endpoints}

In the case of the steepest descent path emerging from an endpoint, we assume that the path is of the form

$$
h_{x}(p)=x+\sum_{j=1}^{\infty} a_{j} p^{j} .
$$

Note that we already incorporated the boundary condition $h_{x}(0)=x$. Substitution into equation (2.1) gives

$$
g\left(x+\sum_{j=1}^{\infty} a_{j} p^{j}\right)=g(x)+i p .
$$

Taking the Taylor expansion of $g$ around $x$ yields the equation

$$
\sum_{k=1}^{\infty} \frac{\left(\sum_{j=1}^{\infty} a_{j} p^{j}\right)^{k}}{k !} g^{(k)}(x)=i p .
$$


The coefficients can now be obtained by series inversion. The first few coefficients are given explicitly by, with evaluation in $x$ implied,

$$
\begin{aligned}
& a_{1}=\frac{i}{g^{\prime}}, \quad a_{2}=\frac{1}{2} \frac{g^{\prime \prime}}{\left(g^{\prime}\right)^{3}}, \\
& a_{3}=\frac{i}{12} \frac{1}{\left(g^{\prime}\right)^{5}}\left(2 g^{\prime} g^{(3)}-\left(g^{\prime \prime}\right)^{2}\right), \\
& a_{4}=-\frac{1}{24} \frac{1}{\left(g^{\prime}\right)^{7}}\left(g^{(4)}\left(g^{\prime}\right)^{2}+10 g^{\prime} g^{\prime \prime} g^{(3)}-15\left(g^{\prime \prime}\right)^{3}\right) .
\end{aligned}
$$

In general, $a_{k}$ is given in terms of derivatives of $g$ up to order $k$.

We define the local path $\tilde{h}_{x}$ by truncating the series of $h_{x}$ after $m$ terms,

$$
\tilde{h}_{x}(p)=x+\sum_{j=1}^{m-1} a_{j} p^{j} .
$$

This means that the left and right hand side of (2.1) match up to order $m$,

$$
g\left(\tilde{h}_{x}(p)\right)=g(x)+i p+\mathcal{O}\left(p^{m}\right), \quad p \rightarrow 0 .
$$

¿From this path we can define the steepest descent integral with an approximated path, using the notation $\tilde{f}_{x}(p)=f\left(\tilde{h}_{x}(p)\right) \tilde{h}_{x}^{\prime}(p)$ and $\tilde{g}_{x}(p)=g\left(\tilde{h}_{x}(p)\right)$,

$$
I\left[f ; \tilde{h}_{x}, P\right]=\int_{0}^{P} \tilde{f}_{x}(p) e^{i \omega \tilde{g}_{x}(p)} \mathrm{d} p .
$$

We shall later evaluate this integral numerically. The numerical approximation will serve as an approximation to the infinite integral $I\left[f ; h_{x}\right]$, we shall see that this is indeed justified in $\S 4.1$.

\subsection{Local paths at stationary points}

We now turn our attention to paths passing through stationary points. Let $x$ be a stationary point of order $r-1$, meaning that $g^{\prime}(x)=\ldots=g^{(r-1)}(x)=0$, but $g^{(r)}(x) \neq 0$. Expanding the path starting at $x$ in integer powers of $p$ is not possible, since $h_{x}(p)$ is singular at $p=0$. This can also be seen from equation (3.2): the first $r-1$ terms in the expansion of $g$ in the left hand side would be zero, which makes it impossible to match the right hand side of the equation. However, proceeding as in $\S 2.2$, the substitution $p=q^{r}$ eliminates this problem. Thus, we assume a path of the form

$$
h_{x}(p)=x+\sum_{j=1}^{\infty} a_{j} p^{j / r} .
$$

Note that the function $h_{x}\left(q^{r}\right)$ is analytic in $q$. Plugging this ansatz into equation (2.1) for the path of steepest descent yields

$$
\sum_{k=r}^{\infty} \frac{\left(\sum_{j=1}^{\infty} a_{j} p^{j / r}\right)^{k}}{k !} g^{(k)}(x)=i p .
$$


The first coefficient is easily obtained,

$$
a_{1}=\sqrt[r]{\frac{i r !}{g^{(r)}(x)}} .
$$

The square root in this expression has $r$ branches in the complex plane, corresponding to the $r$ different paths near the stationary point. More coefficients can be computed recursively. In the case of an order one stationary point, the first four coefficients are, with evaluation in $x$ implied,

$$
\begin{aligned}
& a_{1}= \pm \sqrt{\frac{2 i}{g^{\prime \prime}}}, \quad a_{2}=-\frac{i}{3} \frac{g^{(3)}}{\left(g^{\prime \prime}\right)^{2}}, \\
& a_{3}= \pm \sqrt{\frac{2 i}{g^{\prime \prime}}} \frac{i}{36\left(g^{\prime \prime}\right)^{3}}\left(3 g^{\prime \prime} g^{(4)}-5\left(g^{(3)}\right)^{2}\right), \\
& a_{4}=\frac{1}{270} \frac{1}{\left(g^{\prime \prime}\right)^{5}}\left(40\left(g^{(3)}\right)^{3}-45 g^{(3)} g^{(4)} g^{\prime \prime}+9 g^{(5)}\left(g^{\prime \prime}\right)^{2}\right) .
\end{aligned}
$$

Explicit expressions for the coefficients can be found for general $r$. We refer the reader to [24] for a general description of such explicit expressions.

As in the endpoint case, we form an approximated path by truncating (3.7) after $m$ terms,

$$
\tilde{h}_{x}(p)=x+\sum_{j=1}^{m-1} a_{j} p^{j / r} .
$$

This means that the both sides of (2.1) match up to order $\frac{r+m-1}{r}$,

$$
g\left(\tilde{h}_{x}(p)\right)=g(x)+i p+\mathcal{O}\left(p^{\frac{r+m-1}{r}}\right), \quad p \rightarrow 0 .
$$

This expression agrees with (3.11) for $r=1$. Next, we form the integral

$$
\begin{aligned}
I\left[f ; \tilde{h}_{x}, P\right] & =\int_{0}^{P} \tilde{f}_{x}(p) e^{i \omega \tilde{g}_{x}(p)} \mathrm{d} p . \\
& =\int_{0}^{Q} r q^{r-1} \tilde{f}_{x}\left(q^{r}\right) e^{i \omega \tilde{g}_{x}\left(q^{r}\right)} \mathrm{d} q .
\end{aligned}
$$

with $Q=P^{1 / r}$.

\subsection{Numerical evaluation}

As noted in section $\S 2.2$, it is advantageous to evaluate the half-space integral $I\left[f, h_{x}\right]$ with Gaussian quadrature. Though the integral $I\left[f, \tilde{h}_{x}, P\right]$ is finite, we intend to apply Gaussian half-space quadrature here as well. 
For the numerical evaluation of steepest descent integrals with approximated paths, we rewrite (3.12) as

$$
I\left[f ; \tilde{h}_{x}, P\right]=\int_{0}^{Q} r q^{r-1} \tilde{f}_{x}\left(q^{r}\right) e^{i \omega \tilde{g}_{x}\left(q^{r}\right)+\omega q^{r}} e^{-\omega q^{r}} \mathrm{~d} q .
$$

Note that (3.6) is a special case of (3.13) with $r=1$, so that we can treat the cases of endpoints and stationary points simultaneously. A change of variables $q=\omega^{-1 / r} t$ gives the form

$$
I\left[f ; \tilde{h}_{x}, P\right]=\frac{r}{\omega} \int_{0}^{Q \omega^{1 / r}} t^{r-1} \tilde{f}_{x}\left(t^{r} / \omega\right) e^{i \omega \tilde{g}_{x}\left(t^{r} / \omega\right)+t^{r}} e^{-t^{r}} \mathrm{~d} t .
$$

This integral can be evaluated with the same Gaussian half-space quadrature rules with weight function $e^{-t^{r}}$ that were used on the exact steepest descent integrals. To be precise, if that quadrature rule is given by points $x_{i}$ and weights $w_{i}$, then we propose the approximation

$$
I\left[f ; \tilde{h}_{x}, P\right] \approx Q\left[f ; \tilde{h}_{x}\right]:=\frac{r}{\omega} \sum_{i=1}^{n} w_{i} x_{i}^{r-1} \tilde{f}_{x}\left(\frac{x_{i}^{r}}{\omega}\right) e^{i \omega \tilde{g}_{x}\left(\frac{x_{i}^{r}}{\omega}\right)+x_{i}^{r}} .
$$

We expect that this quadrature rule provides a good approximation to $I\left[f ; \tilde{h}_{x}, P\right]$. This is what we examine next in $\S 4$.

\section{Asymptotic error analysis}

Thus far, we have presented a way of obtaining a numerical approximation of $I\left[f ; \tilde{h}_{x}, P\right]$. We will show first in $\S 4.1$ that this finite saddle-point integral is a good (asymptotic) approximation to the infinite steepest descent integral $I\left[f ; h_{x}\right]$. Next, we shall investigate in $\S 4.2$ the numerical approximation of $I\left[f ; \tilde{h}_{x}, P\right]$ by Gaussian quadrature. Theorem 4.3 gives the asymptotic order of this approximation. Its proof follows in $\S 4.3$ and $\S 4.4$.

\subsection{The error of using truncated approximate paths}

In the method outlined in section $\S 3$, we replaced the exact path of steepest descent $h_{x}$ originating at $x$ with an approximation $\tilde{h}_{x}$ that is valid only near $x$. By our assumptions of analyticity, the path taken does not change the value of the integral. However, since the approximate path may diverge away from the exact path for large $P$, the limit $P \rightarrow \infty$ may result in both paths leading into different sectors of the complex plane. It is clear that the integral along the approximate path should be truncated at finite $P$ to avoid this. In the following theorem and corollary, we prove that the difference between the exact steepest descent integral $I\left[f ; h_{x}\right]$ and the truncated integral $I\left[f ; \tilde{h}_{x}, P\right]$ is exponentially small as $\omega \rightarrow \infty$. This implies that using a numerical approximation of $I\left[f ; \tilde{h}_{x}, P\right]$ is justified. 


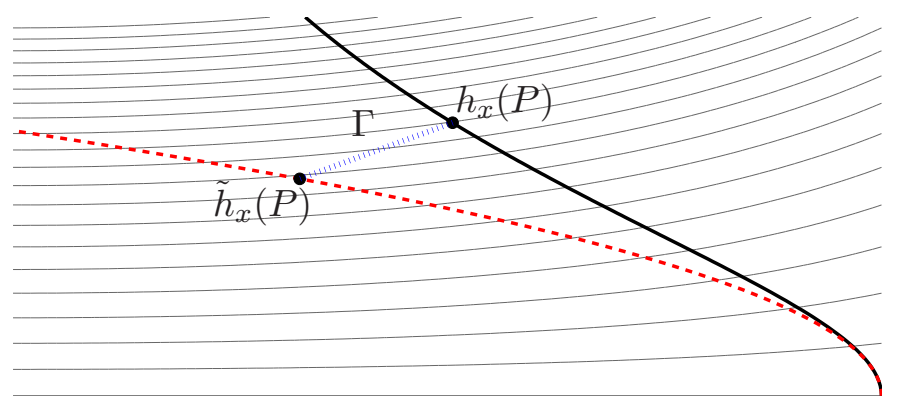

Figure 2: Illustration of the exact (continuous) and approximate (dashed) steepest descent paths. The curve $\Gamma$ connects a truncation of these two paths.

Theorem 4.1. Let $x \in[-1,1]$ be a point of order $r-1$. Assume $f$ and $g$ are analytic, and let $\tilde{h}_{x}(p)$ be an $m$-term approximation to the exact path $h_{x}(p)$ as in (3.10) with $m>1$. Then a constant $P_{0}>0$ exists, such that

$$
I\left[f ; h_{x}, P\right]-I\left[f ; \tilde{h}_{x}, P\right]=\mathcal{O}\left(\omega^{-n}\right), \quad \forall n>0, \quad \forall P<P_{0} .
$$

Proof. By Cauchy's integral theorem we have

$$
I\left[f ; \tilde{h}_{x}, P\right]-I\left[f ; h_{x}, P\right]=\int_{\Gamma} f(s) e^{i \omega g(s)} \mathrm{d} s,
$$

where $\Gamma$ is any simple path connecting $h_{x}(P)$ and $\tilde{h}_{x}(P)$. In the following, we choose $\Gamma$ to be the straight line. We intend to show that the integrand is exponentially small along all of $\Gamma$.

Let us expand $g$ in a Taylor series around $h_{x}(P)$. We have $g(x+\delta)=$ $\mathcal{O}\left(\delta^{r}\right)$ and $g^{(j)}(x+\delta)=\mathcal{O}\left(\delta^{r-j}\right)$. Since $h_{x}(p)=\mathcal{O}\left(p^{1 / r}\right)$, we find that

$$
g^{(j)}\left(h_{x}(p)\right)=\mathcal{O}\left(p^{(r-j) / r}\right) .
$$

We have by construction that $h_{x}(p)-\tilde{h}_{x}(p)=\mathcal{O}\left(p^{m / r}\right)$ and therefore,

$$
\gamma-h_{x}(P)=\mathcal{O}\left(P^{m / r}\right), \quad \gamma \in \Gamma, \quad P \rightarrow 0 .
$$

To conclude, we note that

$$
\begin{aligned}
g(\gamma)= & g\left(h_{x}(P)+\gamma-h_{x}(P)\right)=\sum_{j=0}^{\infty} g^{(j)}\left(h_{x}(P)\right)\left(\gamma-h_{x}(P)\right)^{j} \\
& =g(x)+i P+\mathcal{O}\left(P^{(r-1+m) / r}\right)+\mathcal{O}\left(P^{(r-2+2 m) / r}\right)+\ldots
\end{aligned}
$$

If $m>1$ and if $P$ is sufficiently small, then the term $i P$ dominates the other terms and $g$ has positive imaginary part along $\Gamma$. It follows that the integrand is exponentially small along all of $\Gamma$. 
Corollary 4.2. Under the assumptions of Theorem 4.1

$$
I\left[f ; h_{x}\right]-I\left[f ; \tilde{h}_{x}, P\right]=\mathcal{O}\left(\omega^{-n}\right), \quad \forall n>0, \quad \omega \rightarrow \infty .
$$

Proof. We have

$$
I\left[f ; h_{x}\right]=I\left[f ; h_{x}, P\right]+\int_{P}^{\infty} \psi(q) e^{-\omega q^{r}} \mathrm{~d} q,
$$

where $\psi(q)$ is analytic in $q$. It follows from repeated integration by parts that

$$
I\left[f ; h_{x}\right]-I\left[f ; h_{x}, P\right]=\mathcal{O}\left(\omega^{-n}\right), \quad \forall n>0 \quad \omega \rightarrow \infty .
$$

The result follows from this and Theorem 4.1.

\subsection{Asymptotic error of the numerical approximation}

Since replacing the paths does not lead to a loss in asymptotic order, the order of the overall method relies on the order of the numerical approximation of $I\left[f ; \tilde{h}_{x}, P\right]$. We evaluate the latter by a quadrature rule. A quadrature rule with $n$ points and with order $d$ with respect to the weight function $e^{-x^{r}}$ satisfies the conditions

$$
\int_{0}^{\infty} x^{j} e^{-x^{r}} \mathrm{~d} x=\sum_{k=1}^{n} w_{k} x_{k}^{j}, \quad j=0, \ldots, d-1 .
$$

Using such a rule for the steepest descent integral leads to an asymptotic error of size $\mathcal{O}\left(\omega^{-(d+1) / r}\right)$ [7, Th.2]. When using an approximate path, we have the following result. Note that by integer division $d \backslash \beta$, we mean that the real quantity $d / \beta$ is rounded towards the nearest smaller integer.

Theorem 4.3. Assume $x$ is either a regular point, $r=1$, or a stationary point of order $r-1>0$. An approximation $I\left[f ; \tilde{h}_{x}, P\right]$ to the steepest descent integral $I\left[f ; h_{x}\right]$ is constructed by replacing the path $h_{x}$ with its $m$-term Taylor expansion $\tilde{h}_{x}$, with $m>1$. Let $Q\left[f ; \tilde{h}_{x}\right]$, given by (3.14), denote the approximation to $I\left[f ; \tilde{h}_{x}, P\right]$, obtained through an $n$-point quadrature rule of order d that satisfies the conditions (4.1).

Define $\beta=r+m-1, k=d \backslash \beta$ and $l=d \bmod \beta$. Then

$$
I\left[f ; h_{x}\right]-Q\left[f ; \tilde{h}_{x}\right]= \begin{cases}\mathcal{O}\left(\omega^{-\frac{d+1}{r}+k}\right), & \text { if } l \leq m-1, \\ \mathcal{O}\left(\omega^{-\frac{d+1}{r}+k+\frac{l-(m-1)}{r}}\right), & \text { if } l>m-1,\end{cases}
$$

for $\omega \rightarrow \infty$. In particular, for $r=1$ we have

$$
I\left[f ; h_{x}\right]-Q\left[f ; \tilde{h}_{x}\right]=\mathcal{O}\left(\omega^{-d-1+d \backslash m}\right) .
$$


We can also formulate an upper bound for the exponents in (4.2) that avoids integer arithmetic.

Corollary 4.4. Under the same conditions as in Theorem 4.3, we have

$$
I\left[f ; h_{x}\right]-Q\left[f ; \tilde{h}_{x}\right]=\mathcal{O}\left(\omega^{-\frac{d+1}{r}+\frac{d}{\beta}}\right) .
$$

Proof. For the first case of (4.2), note that $k=d \backslash \beta \leq d / \beta$. For the second case, assume that $l=K+m-1$ with $0<K<r$. Then $k+\frac{l-(m-1)}{r}=$ $k+\frac{K}{r}<k+\frac{K+m-1}{r+m-1}=\frac{d}{\beta}$.

Let us first compare the result of Theorem 4.3 to the result based on using the exact path. One incurs a loss of minimum $k=d \backslash \beta=d \backslash(r+m-1)$. In order to achieve the full order $(d+1) / r$, one should at least have $k=0$, meaning $d<\beta$,

$$
m<d-r+1 \text {. }
$$

Full order is then achieved if $l \leq m-1$, which is always true whenever $r=1$, and more likely to be violated for larger $r$. In the converse case, we have a maximum order loss of one.

Next, we compare to the result based on using a truncated asymptotic expansion. This is more involved. An $s$-term expansion has asymptotic error $\mathcal{O}\left(\omega^{-(s+1) / r}\right)$ and requires the values $g^{(j)}(x), j=0, \ldots, r+s-1[15]$. Using these same values, we can afford $m=s$. The asymptotic expansion also requires the $s$ values $f^{(j)}(x), j=0, \ldots, s-1$. The proposed method requires $f\left(x_{k}\right)$ and $g\left(x_{k}\right)$ for $k=1, \ldots, n$. We choose $n=s$ and continue by counting evaluations of $f$ or any of its derivatives. For the asymptotic expansion, $s$ values of $f$ lead to order $\frac{s+1}{r}$. A Gaussian quadrature rule with respect to the weight function $e^{-x^{r}}$ yields $d=2 s$. By Corollary 4.4, the numerical saddle-point method then yields an order greater than or equal to

$$
\frac{d+1}{r}-\frac{d}{\beta}=\frac{2 s+1}{r}-\frac{2 s}{r+s-1}=\frac{s+1}{r}+\frac{s}{r}\left[\frac{s-(r+1)}{r+s-1}\right]
$$

Thus we are guaranteed to do at least as good as the asymptotic expansion whenever $s \geq r+1$.

Note that in the above, we ignored the evaluations of $g$ in the complex plane. This is justified in a setting where many integrals of the form $I[f]$ need to be evaluated for the same oscillator $g$, for example when computing moments for later use in Filon-type quadrature [15].

Both these calculations show that the proposed method compares well to both the method with exact paths and asymptotic expansions when $r$ is relatively small. In real-life applications we do however not expect to encounter cases with $r$ being large, we will typically have $r=1$ or $r=2$. 


\subsection{Supporting lemmas}

We once again rewrite the integral $I\left[f ; \tilde{h}_{x}, P\right]$ in the following form:

$$
I\left[f, \tilde{h}_{x}, P\right]=e^{i \omega g(x)} \int_{0}^{Q} \tilde{\psi}(q) e^{i \omega R_{r+m-1}(q)} e^{-\omega q^{r}} \mathrm{~d} q,
$$

where $r-1$ is the order of the point $x, R_{\beta}(q)$ is a function of the form

$$
R_{\beta}(q)=q^{\beta} \sum_{j=0}^{\infty} r_{j} q^{j}
$$

and

$$
\tilde{\psi}(q)=r \tilde{f}_{x}\left(q^{r}\right) q^{r-1},
$$

is a smooth function independent of $\omega$. This formulation follows from the construction of the approximate paths. In particular, the form of $R_{r+m-1}$ follows from (3.11) with $p=q^{r}$.

The following lemma is a generalization of Lemma 2.1 in [7]. That lemma characterized the asymptotic order of a scaled quadrature rule applied to a steepest descent integral of the form (1.2). Assume an $n$-point quadrature rule is given that satisfies the conditions (4.1). It was proved in [7] that, for a function $u(x)$ analytic in $x=0$, the quadrature approximation behaves as

$$
\int_{0}^{\infty} u(x) e^{-\omega x^{r}} \mathrm{~d} x-\omega^{-1 / r} \sum_{k=1}^{n} w_{k} u\left(x_{k} \omega^{-1 / r}\right)=\mathcal{O}\left(\omega^{-(d+1) / r}\right) .
$$

Here, we will allow the integrand to depend on $\omega$ in a benign manner and show that the asymptotic order changes in a way that reflects the possible growth or decay of the integrand as a function of $\omega$.

Lemma 4.5. Assume an n-point quadrature rule is given such that conditions (4.1) hold. Let $u(x ; \omega)$ be analytic in $x=0$ with a positive radius of convergence $R$ for each $\omega \geq \omega_{0}$,

$$
u(x ; \omega)=\sum_{j=0}^{\infty} a_{j}(\omega) x^{j}, \quad|x|<R,
$$

and such that $a_{j}=\mathcal{O}\left(\omega^{\gamma_{j}}\right)$ with $\gamma_{j} \in \mathbb{R}$. If $0<P<R$, then

$$
\int_{0}^{P} u(x ; \omega) e^{-\omega x^{r}} \mathrm{~d} x-\omega^{-1 / r} \sum_{k=1}^{n} w_{k} u\left(x_{k} \omega^{-1 / r} ; \omega\right)=\mathcal{O}\left(\omega^{\max _{j} \geq d} \gamma_{j}-\frac{j+1}{r}\right) .
$$


Proof. We have

$$
\int_{0}^{P} u(x ; \omega) e^{-\omega x^{r}} \mathrm{~d} x=\sum_{j=0}^{\infty} a_{j}(\omega) \int_{0}^{P} x^{j} e^{-\omega x^{r}} \mathrm{~d} x .
$$

Using integration by parts, as in the proof of Corollary 4.2, we find that

$$
\int_{0}^{\infty} x^{j} e^{-\omega x^{r}} \mathrm{~d} x-\int_{0}^{P} x^{j} e^{-\omega x^{r}} \mathrm{~d} x=\mathcal{O}\left(\omega^{-m}\right), \quad \forall m \in \mathbb{N} .
$$

Next, it is straightforward to verify that

$$
\int_{0}^{\infty} x^{j} e^{-\omega x^{r}} \mathrm{~d} x-\omega^{-1 / r} \sum_{k=1}^{n} w_{k}\left(x_{k} \omega^{-1 / r}\right)^{j}= \begin{cases}0, & j<d, \\ \mathcal{O}\left(\omega^{-(j+1) / r}\right), & j \geq d\end{cases}
$$

The first case follows from exactness of the quadrature rule for polynomials up to degree $d-1$. The second case follows because both terms in the left hand side have the given size: the integral can be computed explicitly, the summation contains the factor $\omega^{-(j+1) / r}$.

Combining all of the above proves the result. Note that $u(x ; \omega)$ is evaluated in the points $x_{k} \omega^{-1 / r}$ which, for sufficiently large $\omega$, lie in the radius of convergence of $u$.

Finally, we will examine the asymptotic size of functions of the form $e^{\omega \eta(x)}$ and their derivatives. In order to obtain the result, we use a version of Faà di Bruno's formula expressed with integer partitions. A partition of an natural number $n \geq 0$ is a way of writing it as a sum of natural numbers. The number of different ways to do this is the partition number of $n$, denoted $a(n)$. We write a partition $p$ of the integer $n$ as an array $p=\left(p_{1}, p_{2}, \ldots, p_{n}\right)$, where $p_{j}$ is the number of times the integer $j$ occurs in the sum, i.e.,

$$
\sum_{j=1}^{n} j p_{j}=n
$$

See, e.g., [4] for a detailed treatment of partitions and [17] for Faà di Bruno's formula, which we recall in the following Lemma.

Lemma 4.6 (Faà di Bruno's Formula). If $g$ and $f$ are functions that are sufficiently differentiable, then

$$
\begin{aligned}
& \frac{\mathrm{d}^{n}}{\mathrm{~d} x^{n}} g(f(x))= \\
& \quad \sum \frac{n !}{p_{1} ! p_{2} ! \ldots p_{n} !} g^{(k)}(f(x))\left(\frac{f^{\prime}(x)}{1 !}\right)^{p_{1}}\left(\frac{f^{\prime \prime}(x)}{2 !}\right)^{p_{2}} \ldots\left(\frac{f^{(n)}(x)}{n !}\right)^{p_{n}}
\end{aligned}
$$

where the sum is over all partitions $p$ of $n$ with entries $p_{1}, p_{2}, \ldots, p_{m}$, and $k=p_{1}+p_{2}+\ldots+p_{n}$. 
Lemma 4.7. Let $R_{\beta}(q)$ be defined by (4.5) for an integer $\beta>0$. The derivatives of $e^{\omega R_{\beta}(q)}$, evaluated at $q=0$, have an expansion of the form

$$
\left.\frac{\mathrm{d}^{n}}{\mathrm{~d} q^{n}} e^{\omega R_{\beta}(q)}\right|_{q=0}=\sum_{j=0}^{n \backslash \beta} b_{j} \omega^{j}, \quad \omega \rightarrow \infty,
$$

where \denotes integer division.

Proof. It is clear that

$$
R_{\beta}^{(j)}(0)=0, \quad 0 \leq j<\beta .
$$

Using Faà di Bruno's Formula (Lemma 4.6), we have

$$
\begin{aligned}
& \left.\frac{\mathrm{d}^{n}}{\mathrm{~d} q^{n}} e^{\omega R_{\beta}(q)}\right|_{q=0}= \\
& e^{\omega R_{\beta}(0)} \sum \frac{n !}{p_{1} ! p_{2} ! \ldots p_{n} !}\left(\frac{\omega R_{\beta}^{\prime}(q)}{1 !}\right)^{p_{1}}\left(\frac{\omega R_{\beta}^{\prime \prime}(q)}{2 !}\right)^{p_{2}} \ldots\left(\frac{\omega R_{\beta}^{(n)}(q)}{n !}\right)^{p_{n}}= \\
& \sum \frac{n !}{p_{1} ! p_{2} ! \ldots p_{n} !}\left(\frac{\omega R_{\beta}^{(\beta)}(q)}{\beta !}\right)^{p_{\beta}}\left(\frac{\omega R_{\beta}^{(\beta+1)}(q)}{(\beta+1) !}\right)^{p_{\beta+1}} \ldots\left(\frac{\omega R_{\beta}^{(n)}(q)}{n !}\right)^{p_{n}},
\end{aligned}
$$

where the sum is over all partitions $p$ of $n$. The last line follows from equation (4.8). Clearly, each of the terms in this sum is proportional to $\omega^{\sum_{j=\beta}^{n} p_{j}}$. It is also clear that the expansion consists of positive integer powers of $\omega$. To find the dominating term, we maximise the expression $\sum_{j=\beta}^{n} p_{j}$ over the set of all partitions of $n$. It remains only to prove that

$$
\sum_{j=\beta}^{n} p_{j} \leq n \backslash \beta, \quad \forall p \text { partitions of } n
$$

Assume a partition $q$ of $n$ exists such that

$$
\sum_{j=\beta}^{n} q_{j}=n \backslash \beta+M
$$

with $M>0$. From $q$ we can construct another partition $\tilde{q}$ as follows. We let $\tilde{q}_{\beta}=n \backslash \beta+M$ and $\tilde{q}_{j}=0, j>\beta$. It follows from our construction that

$$
\sum_{j=\beta}^{n} j q_{j} \geq \sum_{j=\beta}^{n} j \tilde{q}_{j}=\beta(n \backslash \beta+M)>n .
$$

No matter how we choose $\tilde{q}_{j}$ for $j<\beta$, $\tilde{q}$ can never satisfy the summation property (4.7) and neither can $q$. This proves the result reductio ad absurdum. 
The final lemma concerns the maximal exponent of $\omega$ that may arise in the result of Lemma 4.5.

Lemma 4.8. Assume that $r$ and $\beta$ are integers such that $\beta>r$ and define the sequence

$$
s_{j}=j \backslash \beta-\frac{j}{r} .
$$

For any positive integer $d$, let $k=d \backslash \beta$ and $l=d \bmod \beta$. The maximum of $\left\{s_{j}\right\}_{j=d}^{\infty}$ is

$$
\max _{j \geq d} s_{j}= \begin{cases}k-\frac{d}{r}, & \text { if } l \leq \beta-r, \\ k+1-\frac{(k+1) \beta}{r}, & \text { if } l>\beta-r .\end{cases}
$$

Proof. For the integer division we have the identity

$$
j \backslash \beta=\frac{j}{\beta}-\frac{1}{\beta}(j \bmod \beta) .
$$

This means that

$$
s_{j}=j\left(\frac{1}{\beta}-\frac{1}{r}\right)-\frac{1}{\beta}(j \bmod \beta) .
$$

The first of these terms is decreasing monotonically. The second term is non-increasing, except when the integer part of $j / \beta$ changes. This implies that the largest element in the sequence for $j \geq d$ is either the first element, $s_{d}$, or $s_{n \beta}$ for some integer $n$. In the latter case, we have

$$
s_{n \beta}=n(1-\beta / r),
$$

which again is decreasing. This means that a maximum must occur at the smallest admissible $n$. This is $n=k$, when $d$ is a multiple of $\beta$, and $n=k+1$ otherwise. This leads to $s_{k \beta}=s_{d}$ as above or $s_{(k+1) \beta}$.

¿From the identity (4.9), we find that the corresponding element is either

$$
s_{d}=d \backslash \beta-\frac{d}{r}=k-\frac{d}{r}
$$

or

$$
s_{(k+1) \beta}=(k+1) \beta \backslash \beta-\frac{(k+1) \beta}{r}=k+1-\frac{(k+1) \beta}{r} .
$$

One easily verifies that the former is larger than the latter if $l<\beta-r$. They are equal if $l=\beta-r$. 


\subsection{Proof of Theorem 4.3}

We assembled enough results in $\S 4.3$ to state a short proof of Theorem 4.3. In the following, let $\beta=r+m-1$.

Proof. Leibniz' formula gives the derivatives of the integrand of (4.4) as the sum

$$
\frac{\mathrm{d}^{n}}{\mathrm{~d} q^{n}}\left[\tilde{\psi}(q) e^{i \omega R_{\beta}(q)}\right]=\sum_{j=0}^{n}\left(\begin{array}{c}
n \\
j
\end{array}\right)\left[\frac{\mathrm{d}^{j}}{\mathrm{~d} q^{j}} e^{i \omega R_{\beta}(q)} \frac{\mathrm{d}^{n-j}}{\mathrm{~d} q^{n-j}} \tilde{\psi}(q)\right] .
$$

Lemma 4.7 applied to each of the these terms gives something of the form,

$$
\frac{\mathrm{d}^{n}}{\mathrm{~d} q^{n}}\left[\tilde{\psi}(q) e^{i \omega R_{\beta}(q)}\right]_{q=0}=\sum_{j=0}^{n \backslash \beta} c_{j} \omega^{j} .
$$

Hence, a Taylor series around $q=0$ has coefficients that are $\mathcal{O}\left(\omega^{n \backslash \beta}\right)$.

All conditions of Lemma 4.5 are satisfied and we can conclude that the error of the quadrature approximation is

$$
I\left[f ; \tilde{h}_{x}, P\right]-Q\left[f ; \tilde{h}_{x}\right]=\mathcal{O}\left(\omega^{\max _{j \geq d} j \backslash \beta-(j+1) / r}\right) .
$$

The maximum in the exponent follows from Lemma 4.8, since

$$
j \backslash \beta-(j+1) / r=-1 / r+[j \backslash \beta-j / r]=-1 / r+s_{j},
$$

where $s_{j}$ is defined as in Lemma 4.8. This leads to the stated order (4.2) of the quadrature approximation. The case $l \leq \beta-r=m-1$ follows immediately. For the second case, one can verify that

$$
k+1-\frac{(k+1) \beta}{r}-\frac{1}{r}=-\frac{d+1}{r}+k+\frac{l-(m-1)}{r} .
$$

For $r=1$, the second case does not arise because then $\beta=r+m-1=m$ and the condition $l \leq m-1$ always holds, so the result simplifies.

Thus far we proved the asymptotic error in approximating $I\left[f ; h_{x}, P\right]$. The final result now follows from Corollary 4.2.

\section{$5 \quad$ Numerical experiments}

In this section we will illustrate the use of the method outlined in section $\S 3$ as well as the results regarding the asymptotic error behaviour predicted in Theorem 4.3. 


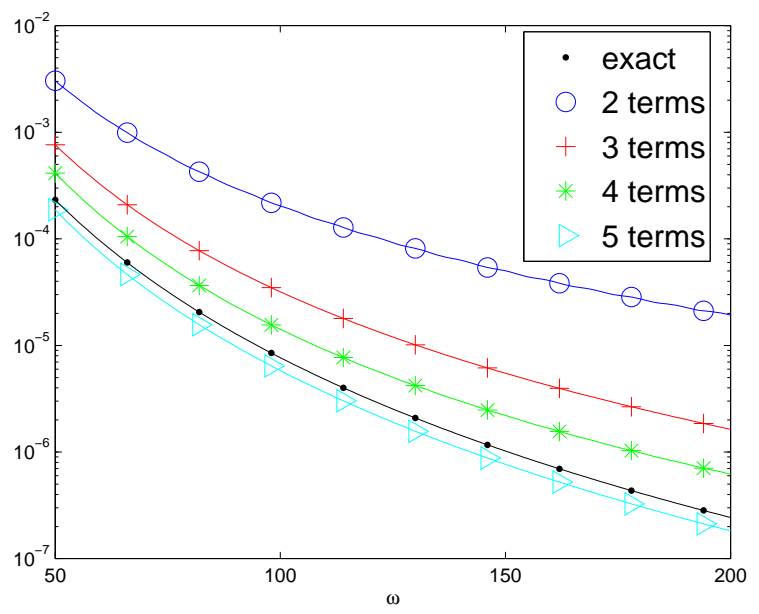

Figure 3: Log-plot of error for different path approximations. Case of regular endpoint.

\subsection{Test of case with no stationary points}

Consider the highly oscillatory integral

$$
I[f]=\int_{-1}^{1} \sin (x) e^{i \omega /(x+2)} \mathrm{d} x
$$

The oscillator $g(x)=1 /(x+2)$ has no stationary points, meaning there are only contributions from the endpoints. The exact paths can be computed in this case.

In Figure 3 we have plotted the error of the two-point Gauss-Laguerre quadrature applied to the resulting line integrals with the given exact paths as well as approximate paths with different number of terms. Note that the approximate paths are constructed only with the knowledge of some derivatives of $g$.

The loss of order when using approximate paths, which can clearly be observed in Figure 3, is predicted in Theorem 4.3. We shall test the conclusion of the theorem by using approximate paths with different number of terms and different number of quadrature points, and then measuring the asymptotic order by regression for each combination. The result of this test can be seen in Table 1 along with the predicted order, $2 n+1-2 n \backslash m$. 


\begin{tabular}{lccccc}
$n \downarrow, m \rightarrow$ & exact & 2 & 3 & 4 & 5 \\
\hline 1 & $3.0(3)$ & $2.0(2)$ & $3.0(3)$ & $3.0(3)$ & $3.0(3)$ \\
2 & $5.0(5)$ & $3.1(3)$ & $4.0(4)$ & $4.1(4)$ & $5.0(5)$ \\
3 & $7.0(7)$ & $4.2(4)$ & $5.1(5)$ & $6.9(6)$ & $6.2(6)$ \\
4 & $9.0(9)$ & $5.3(5)$ & $7.1(7)$ & $7.3(7)$ & $8.0(8)$
\end{tabular}

Table 1: Measured order for different numbers $n$ of Gauss-Laguerre points with $m$ terms in the Taylor expansion of the steepest descent path. First column is with the exact path. Numbers in parentheses are orders predicted in Theorem 4.3.

\subsection{Case of stationary points}

Now consider the integral

$$
I=\int_{0}^{1} \cos (x) e^{i \omega\left(x^{3}+2 x^{2}\right)} \mathrm{d} x,
$$

which has an order one stationary point at the origin. Even in this simple polynomial case the exact path originating from the stationary point is cumbersome to compute. Instead we construct the paths with the coefficients (3.9).

The steepest descent integral corresponding to the path from the stationary point at $x=0$ is computed with a scaled Gaussian quadrature. By using the exact path and a large number of quadrature points, we can nearly eliminate the error contribution from the right endpoint. Thus the error will be dominated by the error from the $x=0$-contribution. Running over a range of different $\omega$ we estimate the order by regression, and the results fit with the predictions from Theorem 4.3 (see Table 2a).

No attempt to use exact paths at the origin was done, and the reference solution was obtained with Matlab's standard quadrature package close to machine precision.

For completion, we include the results from parallel tests done on the integral

$$
I=\int_{0}^{1} e^{i \omega\left(x^{4}+4 x^{3}\right)} \mathrm{d} x,
$$

which has an order 2 stationary point at the origin(Table $2 b$ ).

\section{References}

[1] A. Asheim. A combined Filon/asymptotic quadrature method for highly oscillatory problems. BIT, 48(3):425-448, 2008.

[2] A. Asheim and D. Huybrechs. Local solutions to high frequency 2D scattering problems. Technical report, NTNU, Trondheim, 2008. 


\begin{tabular}{ccccc} 
& 1 & $1.0(1)$ & $1.5(3 / 2)$ & $1.5(3 / 2)$ \\
(a) & 2 & $1.5(3 / 2)$ & $1.5(3 / 2)$ & $2.0(2)$ \\
& 3 & $1.6(3 / 2)$ & $2.4(5 / 2)$ & $2.5(5 / 2)$ \\
& 4 & $1.9(2)$ & $2.4(5 / 2)$ & $3.5(7 / 2)$ \\
& $n \downarrow, m \rightarrow$ & 2 & 3 & 4 \\
\cline { 2 - 4 } (b) & 1 & $0.6(2 / 3)$ & $1.0(1)$ & $1.0(1)$ \\
& 2 & $0.6(2 / 3)$ & $1.0(1)$ & $1.3(4 / 3)$ \\
& 3 & $0.9(1)$ & $1.7(4 / 3)$ & $1.3(4 / 3)$ \\
& 4 & $1.4(1)$ & $1.6(5 / 3)$ & $2.4(2)$
\end{tabular}

Table 2: Measured order for different numbers $n$ of Gauss points with $m$ terms in the expansion of the steepest descent path. Numbers in parentheses are orders predicted in Theorem 4.3. a)-Case of order one stationary point, b)-Case of order two stationary point.

[3] P. Bettess. Short wave scattering, problems and techniques. Phil. Trans. R. Soc. Lond. A, 362:421-443, 2004.

[4] M. Bona. A walk through combinatorics. World scientific co., 2002.

[5] M. Born and E. Wolf. Principles of optics. Cambridge University Press, Cambridge, 1999.

[6] P. J. Davis and P. Rabinowitz. Methods of numerical integration. Computer Science and Applied Mathematics. Academic Press, New York, 1984 .

[7] A. Deaño and D. Huybrechs. Complex Gaussian quadrature of oscillatory integrals. Numer. Math., 2008. To appear.

[8] A. Erdélyi. Asymptotic expansions. Dover publications inc., New York, 1956.

[9] P. Henrici. Applied and computational complex analysis Volume I. Wiley \& Sons, New York, 1974.

[10] D. Huybrechs and S. Olver. Superinterpolation in highly oscillatory quadrature. Technical report. In preparation.

[11] D. Huybrechs and S. Olver. Highly Oscillatory Problems: Computation, Theory and Applications, chapter 2: Highly oscillatory quadrature. Cambridge Univ. Press, 2008.

[12] D. Huybrechs and S. Vandewalle. On the evaluation of highly oscillatory integrals by analytic continuation. SIAM J. Numer. Anal., 44(3):10261048, 2006. 
[13] D. Huybrechs and S. Vandewalle. A sparse discretisation for integral equation formulations of high frequency scattering problems. SIAM J. Sci. Comput., 29(6):2305-2328, 2007.

[14] A. Iserles and S. P. Nørsett. On quadrature methods for highly oscillatory integrals and their implementation. BIT Numer. Math., 44(4):755772, December 2004.

[15] A. Iserles and S. P. Nørsett. Efficient quadrature of highly oscillatory integrals using derivatives. Proc. Roy. Soc. A., 461(2057):1383-1399, 2005.

[16] A. Iserles and S. P. Nørsett. Quadrature methods for multivariate highly oscillatory integrals using derivatives. Math. Comp., 75:1233$1258,2006$.

[17] W. P. Johnson. The curious history of Faà di Bruno's formula. The American Mathematical Monthly, 109(3):217-234, 2002.

[18] D. Levin. Fast integration of rapidly oscillatory functions. J. Comput. Appld. Maths., 67:95-101, 1996.

[19] P. D. Miller. Applied Asymptotic Analysis. American Mathematical Society, Providence, 2006.

[20] F. W. J. Olver. Asymptotics and special functions. Academic Press, Inc, New York, 1974.

[21] S. Olver. Moment-free numerical approximation of highly oscillatory integrals with stationary points. Euro. J. Appl. Maths, 18:435-447, 2006 .

[22] S. Olver. Moment-free numerical integration of highly oscillatory functions. IMA J. of Numer. Anal., 26(2):213-227, Apr. 2006.

[23] S. Olver. On the quadrature of multivariate highly oscillatory integrals over non-polytope domains. Numer. Math., 103(4):643-665, 2006.

[24] J. Wojdylo. Computing the coefficients in Laplace's method. SIAM Rev., 44(1):76-96, 2006.

[25] R. Wong. Asymptotic Approximations of Integrals. SIAM Classics, 2001. 